\title{
LATTICE SEPARATION, COSEPARATION AND REGULAR MEASURES
}

\author{
MAURICE C. FIGUERES \\ The Rockefeller Group \\ 1230 Avenue of the Americas \\ New York, NY 10020-1579
}

(Received November 14, 1994 and in revised form January 10, 1995)

\begin{abstract}
Let $X$ be an arbitrary non-empty set, and let $\mathcal{L}, \mathcal{L}_{1}, \mathcal{L}_{2}$ be lattices of subsets of $X$ containing $\emptyset$ and $X, \mathcal{A}(\mathcal{L})$ designates the algebra generated by $\mathcal{L}$ and $M(\mathcal{L})$, these finite, non-trivial, non-negative finitely additive measures on $\mathcal{A}(\mathcal{L}) . I(\mathcal{L})$ denotes those elements of $M(\mathcal{L})$ which assume only the values zero and one. In terms of a $\mu \in M(\mathcal{L})$ or $I(\mathcal{L})$, various outer measures are introduced. Their properties are investigated. The interplay of measurability, smoothness of $\mu$, regularity of $\mu$ and lattice topological properties on these outer measures is also investigated.

Finally, applications of these outer measures to separation type properties between pairs of lattices $\mathcal{L}_{1}, \mathcal{L}_{2}$ where $\mathcal{L}_{1} \subset \mathcal{L}_{2}$ are developed. In terms of measures from $I(\mathcal{L})$, necessary and sufficient conditions are established for $\mathcal{L}_{1}$ to semi-separate $\mathcal{L}_{2}$, for $\mathcal{L}_{1}$ to separate $\mathcal{L}_{2}$, and finally for $\mathcal{L}_{1}$ to coseparate $\mathcal{L}_{2}$.
\end{abstract}

KEY WORDS AND PHRASES. Outer measures associated with lattice measures, lattice separation and coseparation, weak regularity properties of measures.

1991 AMS SUBJECT CLASSIFICATION CODE. 28C15, $28 \mathrm{~A} 12$.

\section{INTRODUCTION.}

Let $X$ be an arbitrary non-empty set and $\mathcal{L}$ a lattice of subsets of $X$ with $\emptyset, X \in \mathcal{L} . \mathcal{A}(\mathcal{L})$ is the algebra generated by $\mathcal{L}$, and $M(\mathcal{L})$ is the set of finite, non-negative, non-trivial, finitely additive measures on $\mathcal{A}(\mathcal{L}) . I(\mathcal{L})$ consists of those elements of $M(\mathcal{L})$ that just assume the values zero and one. Associated with a $\mu$ in $M(\mathcal{L})$ or $I(\mathcal{L})$, or a $\mu$ in certain subsets of these sets we consider certain outer measures $\mu^{\prime}$, $\tilde{\mu}, \mu^{\prime \prime}$. We use these outer measures to investigate certain weak regularity properties of measures. Also we investigate certain cases in which $\mu^{\prime}=\mu^{\prime \prime}$ on various lattices.

We next consider a pair of lattices $\mathcal{L}_{1}$ and $\mathcal{L}_{2}$ with $\mathcal{L}_{1} \subset \mathcal{L}_{2}$, and we use the above mentioned outer measures to characterize separation properties between $\mathcal{L}_{1}$ and $\mathcal{L}_{2}$. In particular, we investigate in detail necessary and sufficient conditions for $\mathcal{L}_{1}$ to coseparate $\mathcal{L}_{2}$, and for $\mathcal{L}_{1}$ to either separate or semiseparate $\mathcal{L}_{2}$. The material developed here extends the work done in $[4,5,6,8]$.

We begin in Section 2 with a brief review of the notations and some basic terminology used in the paper. We also introduce in this section, the associated outer measures indicated above. Section 3 is devoted to first reviewing some of the basic properties of these outer measures and then to developing new results, in particular pertaining to equality of $\mu^{\prime}$ and $\mu^{\prime \prime}$ on various lattices. Also we extend in this section the work done in $[3,4]$ on various types of weak regularity which can be expressed in terms of $\mu^{\prime}$ and $\mu^{\prime \prime}$. Finally, Section 4 is devoted to a consideration of separation between the two lattices $\mathcal{L}_{1}, \mathcal{L}_{2}$ where $\mathcal{L}_{1} \subset \mathcal{L}_{2}$ in terms of $\mu^{\prime}, \tilde{\mu}$ Several applications of these results as well as of those in Section 3 are given. 


\section{BACKGROUND AND NOTATIONS.}

We consider in this section the notation and some terminology that will be used. We follow standard usage as found, for example, in $[1,2,3,6]$. We will not review all this terminology and notation, but we will only consider those used extensively throughout the paper.

$X$ is an arbitrary non-empty set and $\mathcal{L}$ is a lattice of subsets of $X$ such that $\emptyset, X \in \mathcal{L} . \mathcal{A}(\mathcal{L})$ is the algebra generated by $\mathcal{L}$, and $M(\mathcal{L})$ consists of those non-trivial, finite, non-negative, finitely additive measures on $\mathcal{A}(\mathcal{L}) . \quad M_{\sigma}(\mathcal{L})$ consists of those $\mu \in M(\mathcal{L})$ such that $L_{n} \downarrow \emptyset, L_{n} \in \mathcal{L}$ imply $\mu\left(L_{n}\right) \rightarrow 0$; we refer to these as the $\sigma$-smooth measures on $\mathcal{L}$. In contrast, $M^{\sigma}(\mathcal{L})$ consists of those $\mu \in M(\mathcal{L})$ which are $\sigma$-smooth on $\mathcal{A}(\mathcal{L}) \cdot$ i.e , if $A_{n} \downarrow \emptyset, A_{n} \in \mathcal{A}(\mathcal{L})$ then $\mu\left(A_{n}\right) \rightarrow 0$; in our case, this is of course equivalent to $\mu$ being countably additive. $M_{R}(\mathcal{L})$ consists of those $\mu \in M(\mathcal{L})$ which are $\mathcal{L}$-regular, i.e., $\mu(A)=\sup \{\mu(L) \mid L \subset A, L \in \mathcal{L}\}$ where $A \in \mathcal{A}(\mathcal{L})$, and $M_{R}^{\sigma}(\mathcal{L})=M_{R}(\mathcal{L}) \cap M_{\sigma}(\mathcal{L})$.

If $\mu \in M_{R}(\mathcal{L}) \cap M_{\sigma}(\mathcal{L})$, then it is easy to see that $\mu \in M^{\sigma}(\mathcal{L})$ In the case of measures which just assume the two values zero and one, we denote the above sets: $I(\mathcal{L}), I_{\sigma}(\mathcal{L}), I^{\sigma}(\mathcal{L}), I_{R}(\mathcal{L})$ and $I_{R}^{\sigma}(\mathcal{L})$ respectively.

Throughout $\mathcal{L}^{\prime}\left\{L^{\prime} \mid L \in \mathcal{L}\right\}$ where, in general, for $E \subset X, E^{\prime}=X-E$.

We also denote by $\delta(\mathcal{L})$, the lattice of countable intersections of sets of $\mathcal{L} . \quad \mathcal{L}$ is a delta lattice if $\delta(\mathcal{L})=\mathcal{L}$, i.e., $\mathcal{L}$ is closed under countable intersections

We adhere to the usual lattice-topological terminology such as $\mathcal{L}$ being countably compact (c.c.), disjunctive, normal, countably paracompact (c.p.), etc.

If $\mu \in M(\mathcal{L})$, then for any $E \subset X$, define

$$
\mu^{\prime}(E)=\inf \left\{u\left(L^{\prime}\right) \mid E \subset L^{\prime}, L \in \mathcal{L}\right\} .
$$

It is clear that $\mu^{\prime} \geq 0, \mu^{\prime}(\emptyset)=0, \mu^{\prime}$ is monotone, $\mu^{\prime}(X)=\mu(X)$, and $\mu^{\prime}$ is finitely subadditive. Analogously, we define $\tilde{\mu}$ by replacing in the definition of $\mu^{\prime}$, the covering class by $\mathcal{L} ; \tilde{\mu}$ has the same properties as $\mu^{\prime}$. If we replace the covering class $\mathcal{L}^{\prime}$ in definition $(2.1)$, by $\mathcal{A}(\mathcal{L})$ this gives rise to $\mu^{\bullet}$ which also enjoys the same fundamental properties as $\mu^{\prime}$ and $\tilde{\mu}$.

If $\mu \in M(\mathcal{L})$, then for $E \subset X$, define

$$
\mu^{\prime \prime}(E)=\inf \left\{\sum_{\imath=1}^{\infty} \mu\left(L_{i}^{\prime}\right) \mid E \subset \bigcup_{1}^{\infty} L_{\imath}^{\prime}, L_{\imath} \in \mathcal{L}\right\}
$$

$\mu^{\prime \prime}$ is an outer measure in the usual sense and $\mu^{\prime \prime}(X)=\mu(X)$ if $\mu \in M_{\sigma}(\mathcal{L})$. If $\mu \in I(\mathcal{L})$ and if $\mu \notin I_{\sigma}(\mathcal{L})$, then $\mu^{\prime \prime} \equiv 0$. For this reason, in the two-valued case, when considering $\mu^{\prime \prime}$ we assume $\mu \in I_{\sigma}(\mathcal{L})$.

For the most part we will consider the two-valued case, since our main goal is to characterize separation properties in terms of the above set functions, and for this purpose the two-valued measure suffice. We will, however, indicate extensions to the more general case.

$\mu \in J(\mathcal{L})$ if $\mu \in I(\mathcal{L})$ and $\mu$ is strongly $\sigma$-smooth on $\mathcal{L}$, i.e., if $L_{n} \downarrow L$ where $L_{n}, L \in \mathcal{L}$, then $\mu\left(L_{n}\right) \rightarrow \mu(L)$. We could, of course, consider such measures in the general case, but they will not play a part in our discussion so we restrict our attention to just the two-valued case for this property.

Let $\mu, \nu$ be either measures or outer measures, then we write $\mu \leq \nu(\mathcal{L})$ if $\mu(L) \leq \nu(L)$ for all $L \in \mathcal{L}$.

In this notation, we have, for $\mu \in M(\mathcal{L})$,

$$
\mu \leq \mu^{\prime}(\mathcal{L}) \quad \text { and } \quad \mu=\mu^{\prime}(\mathcal{L}) \quad \text { if and only if } \quad \mu \in M_{R}(\mathcal{L})
$$

We now summarize some known facts (see $[3,4,5])$ which will be used throughout the paper. If $\nu$ is an outer measure (finitely or countably subadditive) we denote by $\mathcal{S}_{\nu}$ the $\nu$-measure sets. 
Then if

$$
\begin{aligned}
\mu \in I(\mathcal{L}), \mathcal{S}_{\mu^{\prime}}= & \{E \subset X \mid E \supset L, L \in \mathcal{L}, \mu(L)=1 \quad \text { or } \\
& \left.E^{\prime} \supset L, L \in \mathcal{L}, \mu(L)=1\right\} .
\end{aligned}
$$

Similarly, we obtain $\mathcal{S}_{\tilde{\mu}}$ by replacing $L$ by $L^{\prime}$ and $\mathcal{L}$ by $\mathcal{L}^{\prime}$ Also,

$$
\begin{aligned}
& \mathcal{S}_{\mu^{\prime \prime}}=\left\{E \subset X \mid E \supset \bigcap_{1}^{\infty} L_{n}, L_{n} \in \mathcal{L}, \mu\left(L_{n}\right)=1\right. \\
& \text { or } \left.E^{\prime} \supset \bigcap_{1}^{\infty} L_{n}, L_{n} \in \mathcal{L}, \mu\left(L_{n}\right)=1\right\} .
\end{aligned}
$$

An outer measure $\nu$ is regular if for any $E \subset X$, there exists an $M \in \mathcal{S}_{\nu}$ such that $E \subset M$ and $\nu(E)=\nu(M)$

Clearly, for $\mu \in I(\mathcal{L}), \mu^{\prime}, \tilde{\mu}$, are regular, and if $\mu \in I_{\sigma}(\mathcal{L}), \mu^{\prime \prime}$ is regular

Furthermore, we have for

$$
\mu \in I_{\sigma}(\mathcal{L}), \mu \leq \mu^{\prime \prime} \leq \mu^{\prime}(\mathcal{L}), \quad \text { and } \quad \mu^{\prime \prime} \leq \mu^{\prime}=\mu\left(\mathcal{L}^{\prime}\right) .
$$

Of course, $\mu^{\prime \prime}(E) \leq \mu^{\prime}(E)$ for all $E \subset X$

$$
\mu \in I_{R}(\mathcal{L}) \quad \text { if and only if } \quad \mu^{\prime} \leq \tilde{\mu} .
$$

These results extend readily to the more general case of $\mu \in M_{\sigma}(\mathcal{L})$ and $\mu \in M_{R}(\mathcal{L})$.

Next, we recall $($ see $[3,4])$ and sketch a proof of the following

THEOREM 2.1. Let $\mathcal{L}_{1}, \mathcal{L}_{2}$ be lattices of subsets of $X$ such that $\mathcal{L}_{1} \subset \mathcal{L}_{2}$ and $\mathcal{L}_{1}$ separates $\mathcal{L}_{2}$ and let $\nu \in I_{R}\left(\mathcal{L}_{2}\right)$ extend $\mu \in I_{R}\left(\mathcal{L}_{1}\right)$.

Then,

a) $\quad \nu$ is $\mathcal{L}_{1}$-regular on $\mathcal{L}_{2}^{\prime}$, and

b) $\quad \nu$ is unique

PROOF. Let $L_{2} \in \mathcal{L}_{2}$ and $\nu\left(L_{2}^{\prime}\right)=1$, then $L_{2}^{\prime} \supset \tilde{L_{2}}$ where $\tilde{L_{2}} \in \mathcal{L}_{2}$ and $\nu\left(\tilde{L_{2}}\right)=1$ since $\nu \in I_{R}\left(\mathcal{L}_{2}\right)$. But $L_{2} \cap \tilde{L_{2}}=\emptyset$, and $\mathcal{L}_{1}$ separates $\mathcal{L}_{2}$; hence there exist $L_{1}, \tilde{L_{1}} \in \mathcal{L}_{1}$ such that $L_{1} \supset L_{2}$, and $\tilde{L_{1}} \supset \tilde{L_{2}}$, and $L_{1} \cap \tilde{L_{1}}=\emptyset$. Therefore, $\nu\left(\tilde{L_{1}}\right)=1$ and $\tilde{L}_{1} \subset L_{1}^{\prime} \subset L_{2}^{\prime}$. This proves a) from which b) follows trivially.

Again, this result can be easily extended to the more general case.

We will extend Theorem 2.1 considerably in the course of our investigation. We will also investigate other relationships between the pair of lattices $\mathcal{L}_{1}$ and $\mathcal{L}_{2}$. Finally, if $\nu \in M\left(\mathcal{L}_{2}\right)$ and if we restrict $\nu$ to $\mathcal{A}\left(\mathcal{L}_{1}\right)$ where $\mathcal{L}_{1} \subset \mathcal{L}_{2}$, we write $\left.\nu\right|_{\mathcal{L}_{1}}$ or simply $\nu \mid$ if the two lattices are fixed in the discussion. Similar notations will be used for the corresponding restrictions of $\nu^{\prime}$ and $\nu^{\prime \prime}$.

\section{BASIC PROPERTIES OF THE OUTER MEASURES}

We will list in this section some known and some new properties and characterizations of the outer measures introduced in Section 2. We will restrict our attention to the zero-one valued case although many results do extend to the more general case; however, our major interest is in separation properties, and for this the special case is enough. We will indicate some extensions in Section 4.

We note first for $\mu \in I(\mathcal{L})$ that $\mu \leq \mu^{\prime}(\mathcal{L})$, and $\mu=\mu^{\prime}$ on $\mathcal{L}^{\prime}$. While for $\mu \in I_{\sigma}(\mathcal{L})$ :

(1) $\mu \leq \mu^{\prime \prime} \leq \mu^{\prime}(\mathcal{L})$, and

(2) $\mu^{\prime \prime} \leq \mu=\mu^{\prime}\left(\mathcal{L}^{\prime}\right)$.

Also, $\mu=\mu^{\prime}(\mathcal{L})$ if and only if $\mu \in I_{R}(\mathcal{L})$.

Continuing we have.

THEOREM 3.1. a) Let $\mu \in I(\mathcal{L})$, then $\mu \in I_{R}(\mathcal{L})$ if and only if $\mu^{\prime} \leq \tilde{\mu}$. 
b) Let $\mathcal{L}$ be delta and normal, and let $\mu \in I_{\sigma}(\mathcal{L})$, then $\mu^{\prime}=\mu^{\prime \prime}(\mathcal{L})$ This result is also true if $\mathcal{L}$ is c.c or if $\mathcal{L}$ is normal and $\mathrm{c} p$

c) For $\mu \in I(\mathcal{L}), \mathcal{S}_{\mu^{\prime}} \cap \mathcal{L}=\left\{L \in \mathcal{L} \mid \mu(L)=\mu^{\prime}(L)\right\}$

d) If $\mu \in J(\mathcal{L})$, then $\mu^{\prime}=\mu^{\prime \prime}(\mathcal{L})$ and, conversely, if $\mu \in I\left(\mathcal{L}^{\prime}\right)$ and if $\mu^{\prime}=\mu^{\prime \prime}\left(\mathcal{L}^{\prime}\right)$, then $\mu \in J(\mathcal{L})$.

PROOF. a) see [5] b), c), d) see [3], and the case where $\mathcal{L}$ is c c is clear. Next, we recall the following well known theorem

THEOREM 3.2. Let $\mathcal{L}_{1}$ and $\mathcal{L}_{2}$ be lattices of subsets of $X$ such that $\mathcal{L}_{1} \subset \mathcal{L}_{2}$.

a) If $\nu \in I_{R}\left(\mathcal{L}_{2}\right)$, then its restriction to $\mathcal{A}\left(\mathcal{L}_{1}\right)$, denoted by $\left.\nu\right|_{\mathcal{L}_{1}} \in I_{R}\left(\mathcal{L}_{1}\right)$ if $\mathcal{L}_{1}$ semi-separates $\mathcal{L}_{2}$

b) Any $\mu \in I_{R}\left(\mathcal{L}_{1}\right)$ can be extended to a $\nu \in I_{R}\left(\mathcal{L}_{2}\right)$, and if $\mathcal{L}_{1}$ separates $\mathcal{L}_{2}$ the regular extension is unique.

PROOF. Both parts are straightforward to prove.

DEFINITION 3.1. Let $\mu \in I(\mathcal{L})$ (or more generally $M(\mathcal{L})$ ), then $\mu$ is said to be:

a) Weakly regular if $\mu\left(L^{\prime}\right)=\sup \left\{\mu^{\prime}(\tilde{L}) \mid \tilde{L} \subset L^{\prime}, \tilde{L} \in \mathcal{L}\right\}$, and where $L \in \mathcal{L}$.

b) Vaguely regular if $\mu \in I_{\sigma}(\mathcal{L})$ and if $\mu\left(L^{\prime}\right)=\sup \left\{\mu^{\prime \prime}(\tilde{L}) \mid \tilde{L} \subset L^{\prime}, \tilde{L} \in \mathcal{L}\right\}$ where $L \in \mathcal{L}$.

The sets of weakly and vaguely regular measures are denoted by $I_{w}(\mathcal{L})$ and $I_{v}(\mathcal{L})$, (respectively $M_{w}(\mathcal{L}), M_{v}(\mathcal{L})$ in the more general case).

We note that if $\mathcal{L}$ is normal, then $I_{w}(\mathcal{L})=I_{R}(\mathcal{L})$. Also, it is not difficult to show that $I_{v}(\mathcal{L}) \subset J(\mathcal{L})$. We now consider some new results and extensions of some of the preceding theorems.

THEOREM 3.3. Let $\mathcal{L}_{1} \subset \mathcal{L}_{2}$ be two lattices of subsets of $X$. Then:

a) Let $\nu \in I_{R}\left(\mathcal{L}_{2}\right)$ extend $\mu \in I\left(\mathcal{L}_{1}\right)$, then $\left.\nu^{\prime}\right|_{\mathcal{L}_{1}}=\left.\mu^{\prime}\right|_{\mathcal{L}_{1}}$ if and only if $\mu \in I_{R}\left(\mathcal{L}_{1}\right)$.

b) Let $\nu \in I_{R}\left(\mathcal{L}_{2}\right)$ be any regular extension of $\mu \in I_{R}\left(\mathcal{L}_{1}\right)$. Then $\nu=\mu^{\prime}\left(\mathcal{L}_{2}\right)$ if and only if $\nu$ is $\mathcal{L}_{1}$-regular on $\mathcal{L}_{2}^{\prime}$. Also, if $\mu \in I_{R}^{\sigma}\left(\mathcal{L}_{1}\right)$, then $\nu=\mu^{\prime}\left(\mathcal{L}_{2}\right)$ implies $\nu \in I_{\sigma}\left(\mathcal{L}_{2}^{\prime}\right)$.

c) If $\nu \in I_{w}\left(\mathcal{L}_{2}\right)$, and if $\mathcal{L}_{1}$ semi-separates $\mathcal{L}_{2}$, then $\mu=\left.\nu\right|_{\mathcal{L}_{1}} \in I_{w}\left(\mathcal{L}_{1}\right)$. The corresponding statement is also true if $\nu \in I_{v}\left(\mathcal{L}_{2}\right)$.

PROOF. a) Clearly, $\nu^{\prime} \leq \mu^{\prime}$ so, in particular, $\nu^{\prime} \leq \mu^{\prime}\left(\mathcal{L}_{1}\right)$. Suppose $\nu^{\prime}=\mu^{\prime}\left(\mathcal{L}_{1}\right)$. Since $\nu=\nu^{\prime}\left(\mathcal{L}_{2}\right)$, we have $\nu=\nu^{\prime}=\mu^{\prime}\left(\mathcal{L}_{1}\right)$ but $\left.\nu\right|_{\mathcal{L}_{1}}=\mu$, hence $\mu=\mu^{\prime}\left(\mathcal{L}_{1}\right)$ and $\mu \in I_{R}\left(\mathcal{L}_{1}\right)$. Conversely, if $\mu \in I_{R}\left(\mathcal{L}_{1}\right)$, then $\mu=\mu^{\prime}\left(\mathcal{L}_{1}\right)$, but $\nu=\nu^{\prime}\left(\mathcal{L}_{2}\right)$ so $\left.\nu^{\prime}\right|_{\mathcal{L}_{1}}=\left.\mu^{\prime}\right|_{\mathcal{L}_{1}}$.

b) For the first part see [4]. If $\mu \in I_{R}^{\sigma}\left(\mathcal{L}_{1}\right)$, let $B_{n}^{\prime} \downarrow \emptyset$, where the $B_{n} \in \mathcal{L}_{2}$. If $\nu\left(B_{n}^{\prime}\right)=1$ for all $n$, then since $\nu$ is $\mathcal{L}_{1}$-regular on $\mathcal{L}_{2}^{\prime}$, there exist $A_{n} \in \mathcal{L}_{1}, A_{n} \subset B_{n}^{\prime}$, and $\mu\left(A_{n}\right)=1$ for all $n$, and since we may assume that the $A_{n} \downarrow \emptyset$, this gives a contradiction.

c) Let $\nu \in I_{w}\left(\mathcal{L}_{2}\right)$, and $\mu=\left.\nu\right|_{\mathcal{L}_{1}}$. If $\mu\left(L_{1}^{\prime}\right)=1, L_{1} \in \mathcal{L}_{1}$, then $\nu\left(L_{1}^{\prime}\right)=1$, and, therefore $L_{1}^{\prime} \supset L_{2} \in \mathcal{L}_{2}$, and $\nu^{\prime}\left(L_{2}\right)=1$ since $\nu \in I_{w}\left(\mathcal{L}_{2}\right)$. By the semi-separation $L_{2} \subset \tilde{L_{1}} \in \mathcal{L}_{1}$ and $L_{1} \cap \tilde{L_{1}}=\emptyset$. Hence $\nu^{\prime}\left(\tilde{L}_{1}\right)=1$, but $\nu^{\prime} \leq \mu^{\prime}$ so $\mu^{\prime}\left(\tilde{L}_{1}\right)=1$ and $\mu \in I_{w}\left(\mathcal{L}_{1}\right)$. A similar proof holds with $\nu \in I_{v}\left(\mathcal{L}_{2}\right)$, and $\mu \in I_{v}\left(\mathcal{L}_{1}\right)$.

DEFINITION 3.2. Let $\mathcal{L}_{1} \subset \mathcal{L}_{2}$ be two lattices of subsets of $X . \mathcal{L}_{2}$ is $\mathcal{L}_{1}$ countably compact (c.c.) if $B \in \mathcal{L}_{2}$, and $B \subset \bigcup_{n=1}^{\infty} A_{n}^{\prime}, A_{n} \in \mathcal{L}_{1}$ implies that there exists a finite subcovering.

Clearly, if $\mathcal{L}_{2}$ if $\mathcal{L}_{1}$ c.c., then $\mathcal{L}_{1}$ is c.c., and if $\mu \in I\left(\mathcal{L}_{1}\right)=I_{\sigma}\left(\mathcal{L}_{1}\right)$, then it is easy to see that $\mu^{\prime}=\mu^{\prime \prime}\left(\mathcal{L}_{2}\right)$. We will return to this property in Section 4 .

THEOREM 3.4. Suppose $\mathcal{L}_{1}$ separates $\mathcal{L}_{2}$, and suppose $\nu_{1}, \nu_{2} \in I_{w}\left(\mathcal{L}_{2}\right)$, both extend $\mu \in I\left(\mathcal{L}_{1}\right)$, then $\nu_{1}=\nu_{2}$.

PROOF. Suppose there exists a $B \in \mathcal{L}_{2}$ such that $\nu_{1}(B)=0$ but $\nu_{2}(B)=1$, then $\nu_{1}\left(B^{\prime}\right)=1$. Hence, there exists $\tilde{B} \in\left(\mathcal{L}_{2}\right), \tilde{B} \subset B^{\prime}$, and $\nu_{1}^{\prime}(\tilde{B})=1$. Now, there exist $A, \tilde{A} \in \mathcal{L}_{1}$ with $B \subset A$, $\tilde{B} \subset \tilde{A}$, and $A \cap \tilde{A}=\emptyset$. Now $\nu_{2}(B)=1$ implies $\nu_{2}(A)=\mu(A)=1$, so $\mu\left(A^{\prime}\right)=0$ or $\nu_{1}\left(A^{\prime}\right)=0$ 
and $A^{\prime} \supset \tilde{B}$, so $\nu_{1}^{\prime}(\tilde{B})=0$, a contradiction. Hence, we must have $\nu_{2} \leq \nu_{1}\left(\mathcal{L}_{2}\right)$. Similarly, we get $\nu_{1} \leq \nu_{2}(\mathcal{L})$ so $\nu_{1}=\nu_{2}$

THEOREM 3.5. Let $\mathcal{L}_{1} \subset \mathcal{L}_{2}$ be lattices of subsets of $X$ such that $\mathcal{L}_{1}$ semi-separates $\mathcal{L}_{2}$ and $\mathcal{L}_{1}$ is a delta lattice. Let $\mu \in I_{\sigma}\left(\mathcal{L}_{1}\right)$, and $\mu^{\prime}=\mu^{\prime \prime}\left(\mathcal{L}_{1}\right)$, then $\mu^{\prime}=\mu^{\prime \prime}\left(\mathcal{L}_{2}\right)$.

PROOF. Of course, $\mu^{\prime \prime} \leq \mu^{\prime}\left(\mathcal{L}_{2}\right)$. Suppose $L_{2} \in \mathcal{L}_{2}$ and $\mu^{\prime}\left(L_{2}\right)=1$, but $\mu^{\prime \prime}\left(L_{2}\right)=0$ Then, there exists $A_{2} \in \mathcal{L}_{1}$ such that $L_{2} \subset \bigcup_{1}^{\infty} A_{\imath}^{\prime}$, and $\mu\left(A_{\imath}^{\prime}\right)=0$, for all $i$. Hence $L_{2}^{\prime} \supset \bigcap_{1}^{\infty} A_{\imath} \in \mathcal{L}_{1} \quad$ Now there exists an $A \in \mathcal{L}_{1}$ such that $L_{2} \subset A$ and $A \cap \bigcap_{1}^{\infty} A_{\imath}=\emptyset$. Consequently, $\mu^{\prime}(A)=1$, thus $\mu^{\prime \prime}(A)=1$, but $A \subset \bigcup_{1}^{\infty} A_{\imath}^{\prime}$, so $\mu^{\prime \prime}(A)=0$, and we are done.

Next we have:

THEOREM 3.6. Let $\mathcal{L}_{1} \subset \mathcal{L}_{2}$ be lattices of subsets of $X$ such that $\mathcal{L}_{1}$ separates $\mathcal{L}_{2}$. If $I_{w}\left(\mathcal{L}_{1}\right)=I_{R}\left(\mathcal{L}_{1}\right)$, then $I_{w}\left(\mathcal{L}_{2}\right)=I_{R}\left(\mathcal{L}_{2}\right)$.

PROOF. Let $\nu \in I_{w}\left(\mathcal{L}_{2}\right)$, then $\mu=\left.\nu\right|_{\mathcal{L}_{1}} \in I_{w}\left(\mathcal{L}_{1}\right)=I_{R}\left(\mathcal{L}_{1}\right)$ by hypothesis and by Theorem 3.3 c). Now let $L_{2} \in \mathcal{L}_{2}$ with $\nu\left(L_{2}^{\prime}\right)=1$. Then, there exists $\tilde{L}_{2} \in \mathcal{L}_{2}$ such that $L_{2}^{\prime} \supset \tilde{L_{2}}$, and $\nu^{\prime}\left(\tilde{L}_{2}\right)=1$. Hence, there exist $L_{1}, \tilde{L}_{1} \in \mathcal{L}_{1}$ with $L_{2} \subset L_{1}$, and $\tilde{L}_{2} \subset \tilde{L}_{1}$, and $L_{1} \cap \tilde{L_{1}}=0$. Hence, $\nu^{\prime}\left(\tilde{L}_{1}\right)=1$, so $\mu^{\prime}\left(\tilde{L}_{1}\right)=1$, and also $\mu\left(\tilde{L_{1}}\right)=1$ since $\mu \in I_{R}\left(\mathcal{L}_{1}\right)$. But $\mu\left(\tilde{L}_{1}\right)=\nu\left(\tilde{L}_{1}\right)$, therefore $\nu \in I_{R}\left(\mathcal{L}_{2}\right)$.

We close this section with the following useful result

THEOREM 3.7. Let $\mu \in I(\mathcal{L})$, then $\mu^{\prime}$ is countably additive on $\mathcal{S}_{\mu^{\prime}}$ if and only if $\mu \in I_{\sigma}(\mathcal{L})$.

PROOF. If $\mu^{\prime}$ is countably additive on $\mathcal{S}_{\mu^{\prime}}$ and if $\mu \notin I_{\sigma}(\mathcal{L})$, then there exist $A_{n} \downarrow \emptyset, A_{n} \in \mathcal{L}$, and $\mu\left(A_{n}\right)=1$ for all $n$, then $\mu^{\prime}\left(A_{n}\right)=1$ for all $n$. But then $A_{n} \in \mathcal{S}_{\mu^{\prime}}$ by Theorem $\left.3.1 \mathrm{c}\right)$. Then $\mu^{\prime}$ is not countably additive on $\mathcal{S}_{\mu^{\prime}}$. Conversely, suppose $\mu \in I_{\sigma}(\mathcal{L})$ then $\mu \leq \mu^{\prime \prime} \leq \mu^{\prime}(\mathcal{L})$, and, for $E \in \mathcal{S}_{\mu^{\prime}}$,

$$
\mu^{\prime}(X)=\mu(X)=\mu^{\prime}(E)+\mu^{\prime}\left(E^{\prime}\right) \geq \mu^{\prime \prime}(E)+\mu^{\prime \prime}\left(E^{\prime}\right) \geq \mu^{\prime \prime}(X)=\mu(X),
$$

thus $E \in \mathcal{S}_{\mu^{\prime \prime}}$ by the regularity of $\mu^{\prime \prime}$ and $\left.\mu^{\prime \prime}\right|_{\mathcal{S}_{\mu^{\prime}}}=\left.\mu^{\prime}\right|_{\mathcal{S}_{\mu^{\prime}}}$, but $\mu^{\prime \prime}$ is countably additive on $S_{\mu^{\prime \prime}}$; hence, $\mu^{\prime}$ must be countably additive on $\mathcal{S}_{\mu^{\prime}}$.

REMARK. Similarly if $\mu \in I(\mathcal{L})$, then $\tilde{\mu}$ is countably additive on $\mathcal{S}_{\tilde{\mu}}$ if and only if $\mu \in I_{\sigma}\left(\mathcal{L}^{\prime}\right)$. This follows by duality between $\mathcal{L}$ and $\mathcal{L}^{\prime}$ and $\mu^{\prime}$ and $\tilde{\mu}$. Clearly, we could make such variations in all our theorems, but we do not since it is a routine matter.

\section{APPLICATIONS OF THE OUTER MEASURES TO SEPARATION PROPERTIES}

We consider mainly in this section two lattices of subsets of $X, \mathcal{L}_{1}$ and $\mathcal{L}_{2}$ such that $\mathcal{L}_{1} \subset \mathcal{L}_{2}$. We will characterize various separation properties between $\mathcal{L}_{1}$ and $\mathcal{L}_{2}$ in terms of the outer measures previously introduced, and then we shall give several applications of these results.

THEOREM 4.1. Let $\mathcal{L}_{1}$ and $\mathcal{L}_{2}$ be lattices of subsets of $X$ such that $\mathcal{L}_{1} \subset \mathcal{L}_{2}$. Let $\mu \in I_{R}\left(\mathcal{L}_{1}\right)$ and let $\nu \in I_{R}\left(\mathcal{L}_{2}\right)$ be an extension of $\mu$. Then a) $\mathcal{L}_{1}$ semi-separates $\mathcal{L}_{2}$ if and only if for every $\mu \in I_{R}\left(\mathcal{L}_{1}\right), \mu^{\prime}=\tilde{\mu}\left(\mathcal{L}_{2}\right)$.

b) $\quad \mathcal{L}_{1}$ separates $\mathcal{L}_{2}$ if and only if for each $\mu \in I_{R}\left(\mathcal{L}_{1}\right), \nu=\tilde{\mu}\left(\mathcal{L}_{2}\right)$.

PROOF. a) The proof of a) is generally known (see $[4,5,6,8])$.

b) In general, we have $\nu \leq \mu^{\prime} \leq \tilde{\mu}\left(\mathcal{L}_{2}\right)$, since $\mu \in I_{R}\left(\mathcal{L}_{1}\right)$ (see (2.5)). Now, suppose $\mathcal{L}_{1}$ separates $\mathcal{L}_{2}$. If $\nu\left(L_{2}\right)=0$, and $\tilde{\mu}\left(L_{2}\right)=1$ where $L_{2} \in \mathcal{L}_{2}$, then $\nu\left(L_{2}^{\prime}\right)=1$. Consequently, there exist an $L_{1} \in \mathcal{L}_{1}, L_{1} \subset L_{2}^{\prime}$, and $\mu\left(L_{1}\right)=1$ by Theorem 2.1. Since $L_{2} \cap L_{1}=\emptyset$, there exists an $\tilde{L_{1}} \in \mathcal{L}_{1}$ such that $L_{2} \subset \tilde{L_{1}}$, and $\tilde{L} \cap L_{1}=\emptyset$. Now clearly $\tilde{\mu}\left(L_{2}\right)=1$ implies $\mu\left(\tilde{L_{1}}\right)=1$; however, $\mu\left(L_{1}\right)=1$, a contradiction. Hence, $\nu=\mu^{\prime}=\tilde{\mu}\left(\mathcal{L}_{2}\right)$.

Conversely, if $\nu=\tilde{\mu}\left(\mathcal{L}_{2}\right)$, then $\nu=\mu^{\prime}\left(\mathcal{L}_{2}\right)$ and $\mu^{\prime}=\tilde{\mu}\left(\mathcal{L}_{2}\right)$. Hence, by Theorem 3.3 b) $\nu$ is $\mathcal{L}_{1}$ regular on $\mathcal{L}_{2}^{\prime}$, and by a) of the present theorem, $\mathcal{L}_{1}$ semi-separates $\mathcal{L}_{2}$. If $\mathcal{L}_{1}$ does not separate $\mathcal{L}_{2}$, then there exist $L_{2}, \tilde{L_{2}} \in \mathcal{L}_{2}$ such that $L_{2} \cap \tilde{L_{2}}=\emptyset$, and $\mathcal{H}=\left\{L_{1} \in \mathcal{L}_{1} \mid L_{1} \supset L_{2}\right.$ or $\left.L_{1} \supset \tilde{L_{2}}\right\}$ has the finite 
intersection property. Hence, there exists a $\mu \in I_{R}\left(\mathcal{L}_{1}\right)$ such that $\mu\left(L_{1}\right)=1$ for all $L_{1} \in \mathcal{H}$ Now, let $\nu \in I_{R}\left(\mathcal{L}_{2}\right)$ be an extension of $\mu$. Since $L_{2}^{\prime} \cap \tilde{L}_{2}^{\prime}=X$, either $\nu\left(L_{2}^{\prime}\right)=1$ or $\nu\left(\tilde{L}_{2}^{\prime}\right)=1 \quad$ If $\nu\left(L_{2}^{\prime}\right)=1$, then there exists $A_{1} \in \mathcal{L}_{1}, A_{1} \subset L_{2}^{\prime}$, and $\mu\left(A_{1}\right)=1 \quad$ Now $A_{1} \cap L_{2}=\emptyset \quad$ Consequently, there exists $B_{1} \in \mathcal{L}_{1}, B_{1} \supset L_{2}$ and $A_{1} \cap B_{1}=\emptyset$. Now $B_{1} \in \mathcal{H}$, whence $\mu\left(B_{1}\right)=1$, and we have a contradiction

Similarly if $\nu\left(\tilde{L}_{2}^{\prime}\right)=1$, and we must have $\mathcal{L}_{1}$ separates $\mathcal{L}_{2}$.

We now consider questions of coseparation of lattices. We first show:

THEOREM 4.2. Let $\mathcal{L}_{1}, \mathcal{L}_{2}$ be lattices of subsets of $X$ such that $\mathcal{L}_{1} \subset \mathcal{L}_{2}$. Also, let $\nu_{1}$, $\nu_{2} \in I_{R}\left(\mathcal{L}_{2}\right)$ with $\mu \leq \nu_{1} \mid\left(\mathcal{L}_{1}\right)$, and $\mu \leq \nu_{2} \mid\left(\mathcal{L}_{1}\right)$ where $\mu \in I(\mathcal{L})$. Then $\mathcal{L}_{1}$ coseparates $\mathcal{L}_{2}$ if and only if $\nu_{1}=\nu_{2}$.

PROOF. Suppose $\mathcal{L}_{1}$ coseparates $\mathcal{L}_{2}$. If $\nu_{1} \neq \nu_{2}$, then there exist $B_{1}, B_{2} \in \mathcal{L}_{2}$ such that $\nu_{1}\left(B_{1}\right)=1, \nu_{2}\left(B_{1}\right)=0, \nu_{1}\left(B_{2}\right)=0, \nu_{2}\left(B_{2}\right)=1$, and $B_{1} \cap B_{2}=\emptyset$. Hence, there exist $A_{1}, A_{2} \in \mathcal{L}_{1}$ with $B_{1} \subset A_{1}^{\prime}, B_{2} \subset A_{2}^{\prime}$ and $A_{1}^{\prime} \cap A_{2}^{\prime}=\emptyset$. Hence either $\mu\left(A_{1}\right)=1$ or $\mu\left(A_{2}\right)=1$. If $\mu\left(A_{1}\right)=1$, then $\mu\left(A_{1}^{\prime}\right)=0$ so $\nu_{1}\left(A_{1}^{\prime}\right)=0$, and, therefore $\nu_{1}\left(B_{1}\right)=0$, a contradiction. Similarly, if $\mu\left(A_{2}\right)=1$, thus we must have $\nu_{1}=\nu_{2}$.

Conversely, suppose the condition of the theorem holds. If $\mathcal{L}_{1}$ does not coseparate $\mathcal{L}_{2}$, then there exist $B_{1}, B_{2} \in \mathcal{L}_{2}$ such that $B_{1} \cap B_{2}=\emptyset$, and

$$
W=\left\{A^{\prime} \in \mathcal{L}_{1}^{\prime} \mid B_{1} \subset A^{\prime} \text { or } B_{2} \subset A^{\prime}\right\}
$$

has the finite intersection property. Hence, there exists a $\mu \in I\left(\mathcal{L}_{1}\right)$ such that $\mu\left(A^{\prime}\right)=1$ if $A^{\prime} \supset B_{1}$, or $A^{\prime} \supset B_{2}$. Now, if $\mu(L)=1$ where $L \in \mathcal{L}_{1}$, then $\mu\left(L^{\prime}\right)=0$. Hence, $\left\{L \cap B_{1} \mid \mu(L)=1, L \in \mathcal{L}_{1}\right\}$ has the finite intersection property and $\left\{L \cap B_{2} \mid \mu(L)=1, L \in \mathcal{L}_{1}\right\}$ has also the finite intersection property. Therefore, there exist $\nu_{1}, \nu_{2} \in I_{R}\left(\mathcal{L}_{2}\right)$ with $\nu_{1}\left(B_{1}\right)=1$ and $\nu_{2}\left(B_{2}\right)=1$. But $B_{1} \cap B_{2}=\emptyset$. Consequently $\nu_{1} \neq \nu_{2}$, a contradiction. Hence $\mathcal{L}_{1}$ coseparates $\mathcal{L}_{2}$.

THEOREM 4.3. Let $\mu \in I\left(\mathcal{L}_{1}\right)$, and suppose $\mathcal{L}_{1} \subset \mathcal{L}_{2}$ where $\mathcal{L}_{1}$ coseparates $\mathcal{L}_{2}$. If $\mu \leq \nu\left(\mathcal{L}_{1}\right)$ where $\nu \in I_{R}\left(\mathcal{L}_{2}\right)$, then if $\nu\left(L_{2}^{\prime}\right)=1, L_{2} \in \mathcal{L}_{2}$, there exists an $L_{1} \in \mathcal{L}_{1}, L_{1} \subset L_{2}^{\prime}$, and $\mu\left(L_{1}\right)=1$.

PROOF. $\nu\left(L_{2}^{\prime}\right)=1 \Rightarrow L_{2}^{\prime} \supset \tilde{L}_{2} \in \mathcal{L}_{2}$, and $\nu\left(\tilde{L}_{2}\right)=1$. Now, by coseparation, there exist $L_{1}$, $\tilde{L}_{1} \in \mathcal{L}_{1}$ such that $L_{2} \subset L_{1}^{\prime}, \tilde{L_{2}} \subset \tilde{L}_{1}^{\prime}$ and $L_{1}^{\prime} \cap \tilde{L}_{1}^{\prime}=\emptyset$. Hence, $\tilde{L}_{2} \subset \tilde{L}_{1}^{\prime} \subset L_{1} \subset L_{2}^{\prime}$.

Then $\nu\left(\tilde{L_{2}}\right)=1 \Rightarrow \nu\left(\tilde{L_{1}^{\prime}}\right)=1$, therefore $\mu\left(\tilde{L}_{1}^{\prime}\right)=1$. Consequently $\mu\left(L_{1}\right)=1$.

Finally, we have:

THEOREM 4.4. Let $\mathcal{L}_{1} \subset \mathcal{L}_{2}$ be lattices of subsets of $X$. Let $\nu \in I_{R}\left(\mathcal{L}_{2}\right)$, and $\mu \leq \nu\left(\mathcal{L}_{1}\right)$ where $\mu \in I\left(\mathcal{L}_{1}\right)$. Then $\nu=\nu^{\prime}=\mu^{\prime}\left(\mathcal{L}_{2}\right)$ if and only if $\mathcal{L}_{1}$ coseparates $\mathcal{L}_{2}$.

PROOF. Suppose $\mathcal{L}_{1}$ coseparates $\mathcal{L}_{2}$. Now clearly $\nu=\nu^{\prime}\left(\mathcal{L}_{2}\right)$ since $\nu \in I_{R}\left(\mathcal{L}_{2}\right)$, and, of course, $\nu^{\prime} \leq \mu^{\prime}$. Suppose $\nu\left(L_{2}\right)=0$, where $L_{2} \in \mathcal{L}_{2}$, then $\nu\left(L_{2}^{\prime}\right)=1$, and, therefore, by Theorem 4.3, $L_{2}^{\prime} \supset L_{1} \in \mathcal{L}_{1}$, and $\mu\left(L_{1}\right)=1$, or $L_{2} \subset L_{1}^{\prime}$, and $\mu\left(L_{1}^{\prime}\right)=0$. Hence $\mu^{\prime}\left(L_{2}\right)=0$. Consequently $\nu=\nu^{\prime}=\mu^{\prime}\left(\mathcal{L}_{2}\right)$.

Conversely, assuming the condition of theorem holds, then suppose $\nu_{1}, \nu_{2} \in I_{R}\left(\mathcal{L}_{2}\right)$ and $\mu \leq \nu_{1} \mid\left(\mathcal{L}_{1}\right)$ and $\mu \leq \nu_{2} \mid\left(\mathcal{L}_{1}\right)$, then $\nu_{1}=\nu_{1}^{\prime}=\mu^{\prime}\left(\mathcal{L}_{2}\right)$ and $\nu_{2}=\nu_{2}^{\prime}=\mu^{\prime}\left(\mathcal{L}_{2}\right)$. Therefore, $\nu_{1}=\nu_{2}$, and, by Theorem $4.2, \mathcal{L}_{1}$ coseparates $\mathcal{L}_{2}$.

If $\nu \in I_{R}^{\sigma}\left(\mathcal{L}_{2}\right)$, we can prove even more.

THEOREM 4.5. Let $\mathcal{L}_{1} \subset \mathcal{L}_{2}$ and suppose $\mathcal{L}_{1}$ coseparates $\mathcal{L}_{2}$. If $\nu \in I_{R}^{\sigma}\left(\mathcal{L}_{2}\right)$ and if $\mu<\nu\left(\mathcal{L}_{1}\right)$ where $\mu \in I\left(\mathcal{L}_{1}\right)$, then $\nu^{\prime \prime}=\mu^{\prime \prime}\left(\mathcal{L}_{2}\right)$.

PROOF. Clearly $\mu \in I_{\sigma}\left(\mathcal{L}_{1}\right)$. Also $\nu=\mu^{\prime}\left(\mathcal{L}_{2}\right)$ by Theorem 4.4 .

But $\nu \leq \nu^{\prime \prime}\left(\mathcal{L}_{2}\right)$ and $\nu^{\prime \prime} \leq \mu^{\prime \prime}<\mu^{\prime}$. Therefore, clearly $\nu^{\prime \prime}=\mu^{\prime \prime}\left(\mathcal{L}_{2}\right)$.

REMARK. A number of our results extend to the more general case of measures in $M(\mathcal{L})$. We will not pursue these matters in detail here, but just indicate a few of these extensions: 
1) If $\mathcal{L}_{1} \subset \mathcal{L}_{2}$ are lattices of subsets of $X$, and if $\nu \in M_{R}\left(\mathcal{L}_{2}\right)$ extends $\mu \in M_{R}\left(\mathcal{L}_{1}\right)$, then $\nu=\mu^{\prime}\left(\mathcal{L}_{2}\right)$ if and only if $\nu$ is $\mathcal{L}_{1}$-regular on $\mathcal{L}_{2}^{\prime}$.

2) If $\nu=\mu^{\prime}\left(\mathcal{L}_{2}\right)$ where $\nu \in M_{R}\left(\mathcal{L}_{2}\right)$ is any regular extension of $\mu \in M_{R}\left(\mathcal{L}_{1}\right)$, then $\nu$ is unique. Clearly, this condition is equivalent (since $\mu \in M_{R}\left(\mathcal{L}_{1}\right)$ ) to $\nu=\mu^{\cdot}\left(\mathcal{L}_{2}\right)$ where for $E \subset X$, $\mu^{\bullet}(E)=\inf \{\mu(A) \mid E \subset A, A \in \mathcal{A}(\mathcal{L})\}$.

We note for completeness that the converse of this is known, namely, if for $\mu \in M_{R}\left(\mathcal{L}_{1}\right)$ the regular extension $\nu \in M_{R}\left(\mathcal{L}_{2}\right)$ is unique, then $\nu=\mu^{\cdot}\left(\mathcal{L}_{2}\right)$ (see [7])

3) If $\mathcal{L}_{1} \subset \mathcal{L}_{2}$ and if $\mathcal{L}_{1}$ semi-separates $\mathcal{L}_{2}$, then for $\mu \in M_{R}\left(\mathcal{L}_{1}\right), \tilde{\mu}=\mu^{\prime}\left(\mathcal{L}_{2}\right)$.

We conclude this section by indicating just a few of the applications of our previous results

THEOREM 4.6. Let $\mathcal{L}_{1} \subset \mathcal{L}_{2}$, and suppose $\mathcal{L}_{1}$ separates $\mathcal{L}_{2}$ and let $\mu \in I_{R}^{\sigma}\left(\mathcal{L}_{1}\right)$ be extended to $\nu \in I_{R}\left(\mathcal{L}_{2}\right)$. If a) $\mathcal{L}_{1}$ is delta and normal, or if b) $\mathcal{L}_{2}$ is $\mathcal{L}_{1}$ countably compact, then $\nu=\mu^{\prime}=\mu^{\prime \prime}\left(\mathcal{L}_{2}\right)$, and $\nu \in J\left(\mathcal{L}_{2}^{\prime}\right)$.

PROOF. In case b), $\mu^{\prime}=\mu^{\prime \prime}\left(\mathcal{L}_{2}\right)$ by the remark after Definition 3.2, while in case a) we get $\mu^{\prime}=\mu^{\prime \prime}\left(\mathcal{L}_{2}\right)$ by Theorems 3.5 and 3.1 part b), and, in both cases, $\nu=\mu^{\prime}\left(\mathcal{L}_{2}\right)$ since $\mathcal{L}_{1}$ separates $\mathcal{L}_{2}$.

Finally, we observe that $\nu=\mu^{\prime}\left(\mathcal{L}_{2}\right)$ implies $\nu \in I_{\sigma}\left(\mathcal{L}_{2}^{\prime}\right)$ by Theorem 3.3 part b).

Now let $B_{n} \uparrow B$, where $B_{n}, B \in \mathcal{L}_{2}$, then $\mu^{\prime \prime}\left(B_{n}\right) \uparrow \mu^{\prime \prime}(B)$ since $\mu^{\prime \prime}$ is a regular outer measure from which it follows that $\nu \in J\left(\mathcal{L}_{2}^{\prime}\right)$.

THEOREM 4.7. Let $\mathcal{L}_{1} \subset \mathcal{L}_{2}$ where $\mathcal{L}_{1}$ and $\mathcal{L}_{2}$ are lattices of subsets of $X$. If $\mathcal{L}_{2}$ is $\mathcal{L}_{1}$ countably compact, and if $\mathcal{L}_{1}$ is complement generated and normal, then $\mathcal{L}_{1}$ semi-separates $\mathcal{L}_{2}$.

PROOF. Let $\mu \in I_{R}\left(\mathcal{L}_{1}\right)$, then $\mu^{\prime} \leq \tilde{\mu}$ by (2.5). Suppose $\mu^{\prime}\left(L_{2}\right)=0$ where $L_{2} \in \mathcal{L}_{2}$. Then, there exists $L_{1} \in \mathcal{L}_{1}$, with $L_{2} \subset L_{1}^{\prime}$, and $\mu\left(L_{1}^{\prime}\right)=0$.

But $L_{1}=\bigcap_{n=1}^{\infty} A_{n}^{\prime}, A_{n} \in \mathcal{L}_{1}$ so $L_{1}^{\prime}=\bigcup_{n=1}^{\infty} A_{n}$. By normality of $\mathcal{L}_{1}, L_{1} \subset C_{n}^{\prime} \subset B_{n} \subset A_{n}^{\prime}$ with $C_{n}, B_{n} \in \mathcal{L}_{1}$. Thus, $L_{2} \subset \bigcup_{n=1}^{\infty} A_{n} \subset \bigcup_{n=1}^{n=1} B_{n}^{\prime} \subset \bigcup_{n=1}^{\infty} C_{n} \subset L_{1}{ }^{\prime}$ Hence, $L_{2} \subset \bigcup B_{n,}^{\prime} \subset \bigcup C_{n_{1}}$, where the union is over a finite number of indices. Let $C=\bigcup C_{n_{1}}$, then $C \in \mathcal{L}_{1}$ and $\mu(C)=0$. Hence $\tilde{\mu}\left(L_{2}\right)=0$, a contradiction.

Consequently, $\mu^{\prime}=\tilde{\mu}\left(\mathcal{L}_{2}\right)$, and by Theorem $4.1, \mathcal{L}_{1}$ semi-separates $\mathcal{L}_{2}$.

ACKNOWLEDGMENT. The author wishes to express his appreciation to the referees for correcting a number of statements and consequently making the paper more readable.

\section{REFERENCES}

[1] ALEXANDROFF, A.D., Additive set functions in abstract spaces, Mat. Sb. (N.S.) 8, 50 (1940), 307-348.

[2] BACHMAN, G. and SULTAN, A., Regular lattice measures: mappings and spaces, Pacific $J$. Math. 67, 2 (1976), 291-321.

[3] GRASSI, P., Outer measures and associated lattice properties, Inter. J. Math. \& Math. Sci., 16, 4 (1993), 687-694.

[4] HAJALLIE, K., Lattice measures and separation (to appear).

[5] SZETO, M., On separation of lattices, Inter. J. Math. \& Math. Sci., 14, 2 (1991), 325-338.

[6] SZETO, M., On normal lattices and separation properties of lattices, Jour. Ind. Math. Soc., 58, 1 (1992), 51-64.

[7] TARASHCHANSKII, M.T., Uniqueness of the extension of a regular measure (Russian) Ukrainskii Matematicheskii Zhurnal 41 (1989), 3, 372-377, Translation in Ukranian Math. J. 41 (1989), 3, 329-333.

[8] VLAD, C., On normal lattices and semi-separation of lattices, Jour. Ind. Math. Soc. 56 (1991), 259-273. 


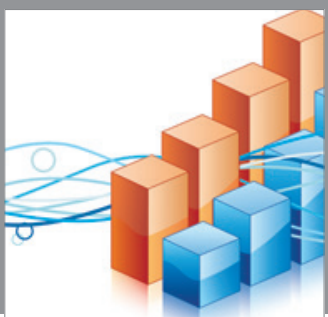

Advances in

Operations Research

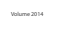

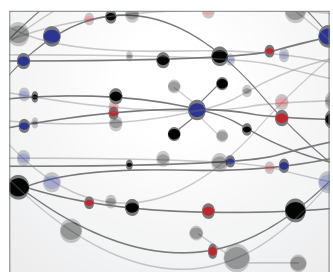

\section{The Scientific} World Journal
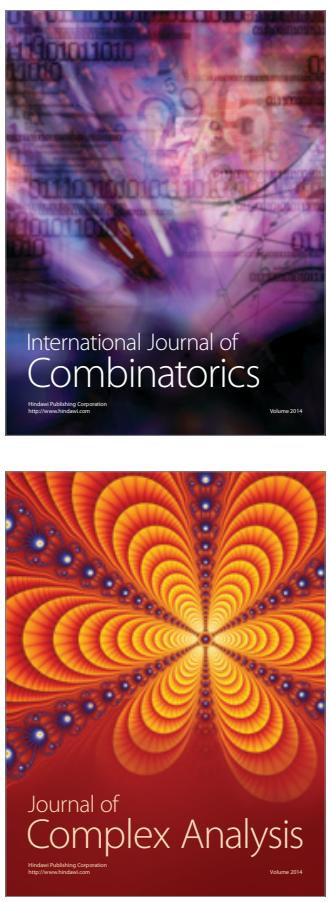

International Journal of

Mathematics and

Mathematical

Sciences
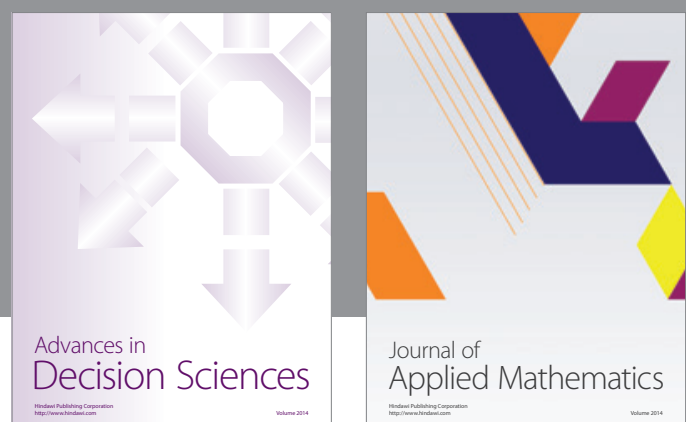

Journal of

Applied Mathematics
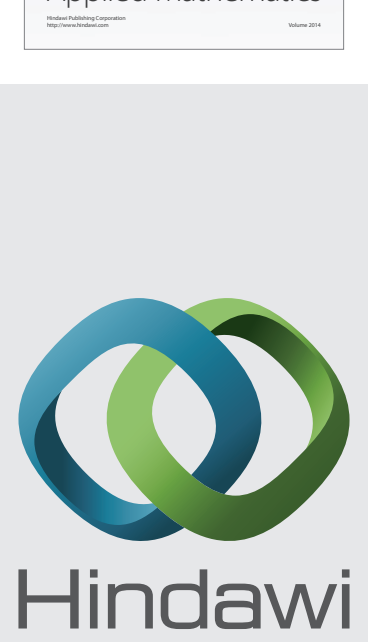

Submit your manuscripts at http://www.hindawi.com
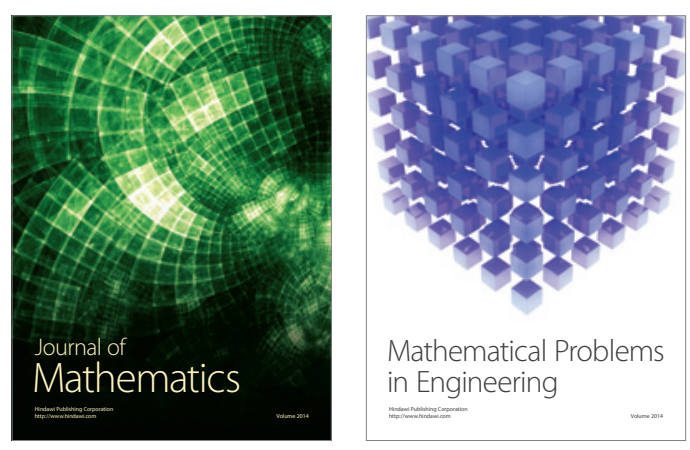

Mathematical Problems in Engineering
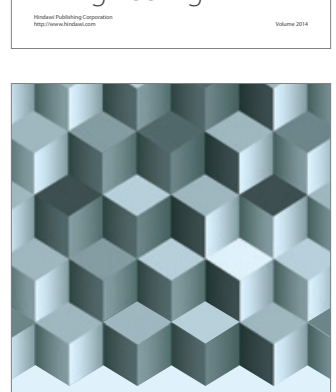

Journal of

Function Spaces
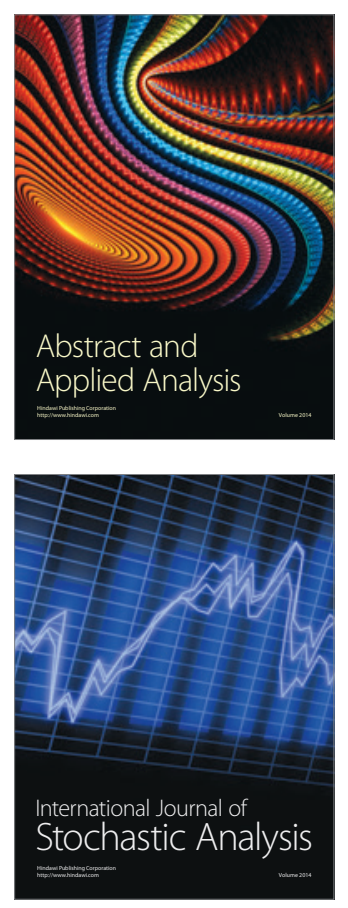

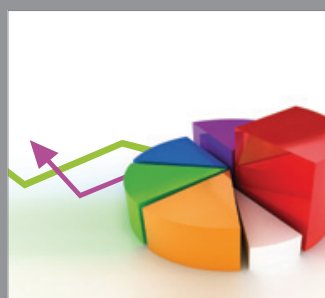

ournal of

Probability and Statistics

Promensencen
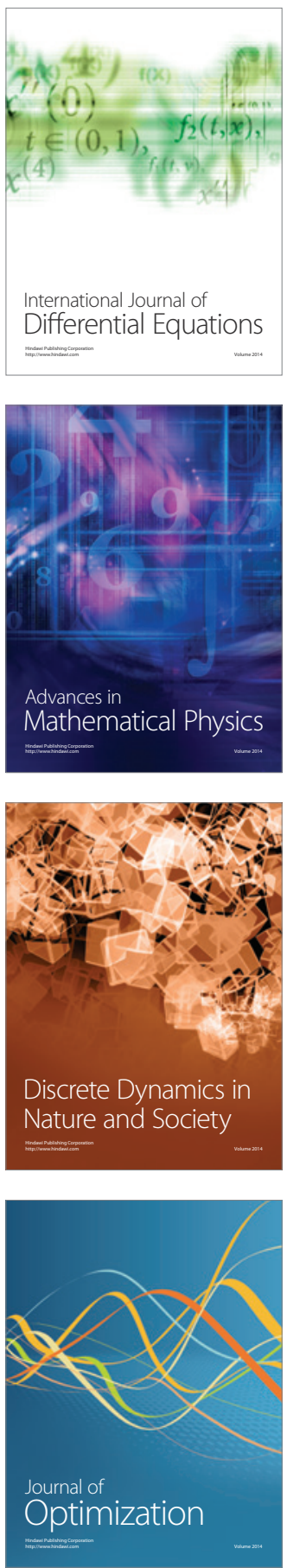